\title{
Editorial: Introduction to the 36th Annual Gallery of Fluid Motion and Summary of 19 Years of Winners (Atlanta, Georgia, USA 2018)
}

\author{
David Hu, ${ }^{1}$ Brian Thurow, ${ }^{2}$ Ken Kiger, ${ }^{3}$ and Alexander Lee ${ }^{1}$ \\ ${ }^{1}$ School of Biological Sciences, Georgia Institute of Technology, Atlanta, Georgia 30332, USA \\ ${ }^{2}$ Department of Aerospace Engineering, Auburn University, Auburn, Alabama 36849, USA \\ ${ }^{3}$ Department of Mechanical Engineering, University of Maryland, College Park, Maryland 20742, USA
}

(Received 16 October 2019; published 24 October 2019)

DOI: 10.1103/PhysRevFluids.4.100002

The 36th Gallery of Fluid Motion (GFM) was held November 18-20, 2018, during the 71st annual meeting of the Division of Fluid Dynamics of the American Physical Society (APS) in Atlanta, Georgia. The gallery received submissions in two categories, posters and videos, all of which are available on the Gallery's website (http://gfm.aps.org/meetings/dfd-2018). In total, 56 posters and 80 videos were received from 20 countries. The content ranged over a broad spectrum of fluid mechanics topics, with the largest number of entries (26\%) on the subject of drops and bubbles. The popularity of this topic is in line with the prevalence of winning entries from 2000 to the current year, as shown in Fig. 1. Historically, the category that won the most was drops, jets and splashes, which accounted for $17 \%$ of all winning entries. This is likely due to the range of rich behavior these flows produce combined with high-contrast images provided by the dynamic gas/liquid interface. Other categories that scored wins included bubbles, capillary flows, vortices and turbulence.

The winning videos were selected by an esteemed panel of 20 judges from the US, Korea, Australia, France, and UK. The composition of the panel consisted of $30 \%$ women or underrepresented groups, as well as a number of previous Gallery of Fluid Motion Winners. Each video was scored by three randomly assigned judges, who gave scores ranging from 0 to 100 . The top 12 video entries were scored an additional 14 times.

Given that the 80 video submissions required 240 different judging sessions, one of us (Alex Lee) automated the process of assigning the entries using a custom Python script. With 20 judges, each judge would have to evaluate an average of 12 submissions. Therefore, each judge's name was placed in a metaphorical hat 13 times and selected without replacement to judge each of the submissions, checking to ensure that a judge was not assigned to the same submission multiple times. Each judge recorded their evaluations on Google Spreadsheets and scores were compiled through Python, using the Google Sheets API. Given the large number of entries, we recommend that future committees should involve someone with coding experience. We have passed on our code to the 2020 Gallery of Fluid Motion organizers.

The winning poster entries were selected by an esteemed panel of 7 judges with representation from the United States, the international community and women. Each judge viewed every poster and submitted scores ranging from 0 to 5 for posters that they deemed as competitive for an award. After removing the minimum and maximum scores for each poster, the remaining scores were averaged, resulting in the ranked list of the Top 8 posters.

Judging the overall winners of the Gallery was particularly difficult due to the high quality of the entries. The top 12 entries were discussed by a panel composed of the authors of this article. In this manner, five winning posters and seven winning videos were identified. The top three winners in each category were designated the Milton van Dyke Award winners. The winning GFM posters 


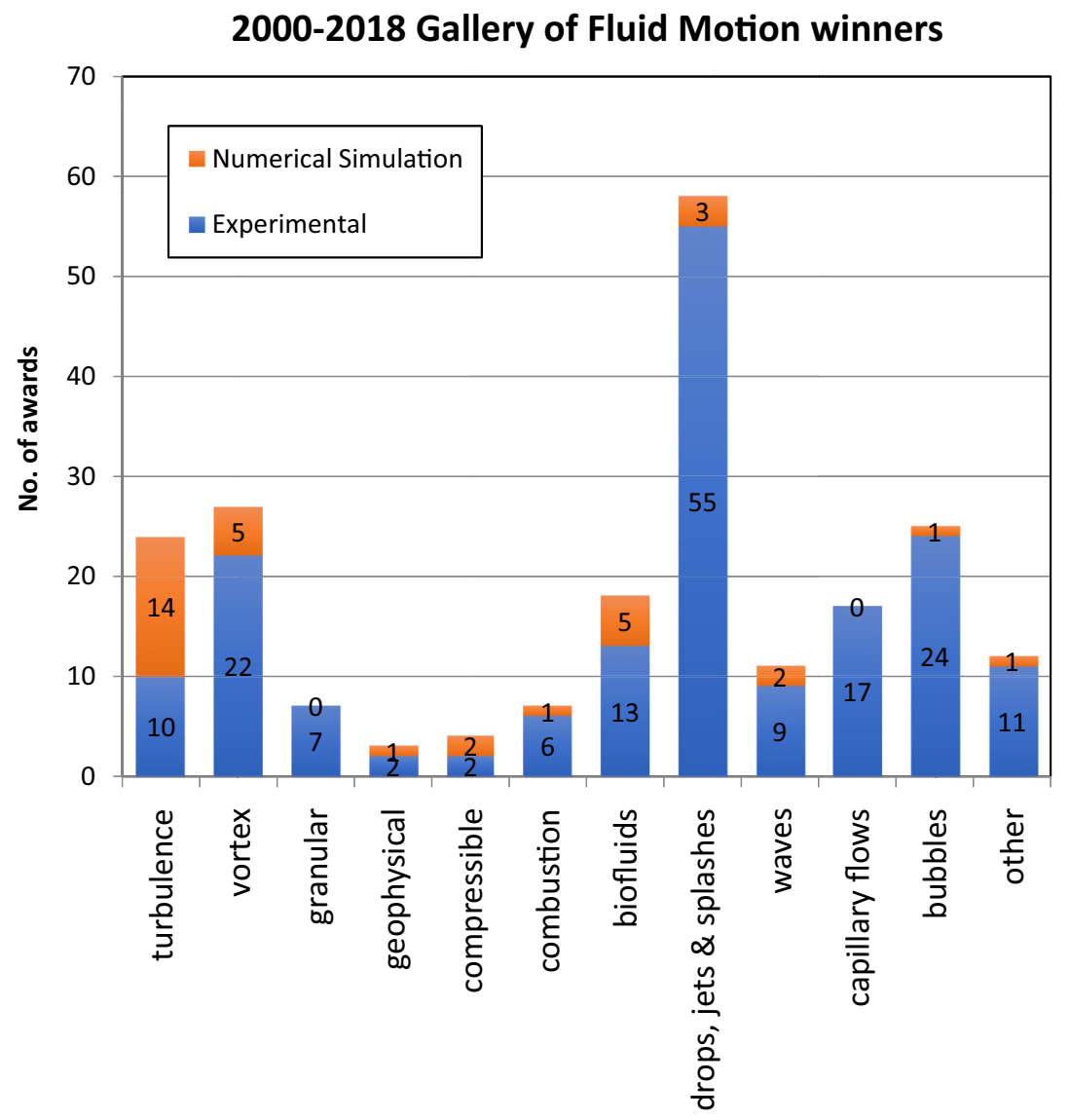

FIG. 1. The number of Gallery of Fluid Motion awards from 2000 to 2018, sorted by the different categories of submission. Total number of winning entries over this period is $\mathrm{N}=225$. Data collected by Ken Kiger.

and videos are listed below. The participants in the Video Gallery were interested in their scores. In order to encourage top participants to resubmit next year, we emailed all the participants a list of the entries that obtained better than average scores.

A special thanks to Paul Dlug of APS who coordinated giving all our judges electronic access to the entries, and to Peggy Holland of Meetings and More for keeping us on top of deadlines.

Videos (7 winners: 3 Milton van Dyke and 4 Gallery Winners)

\section{Milton van Dyke Video Award winners}

(1) V0054 Shaky life of a water drop in an anise oil-rich environment. Óscar R. Enríquez, Daniel Robles, Pablo Peñas-López, Javier Rodríguez-Rodríguez Universidad Carlos III de Madrid; Universidad Nacional Autónoma de México

(2) V0018 Premixed-flame oscillations in narrow channels.

Daniel Martínez-Ruiz, Fernando Veiga-López, Mario Sánchez-Sanz Universidad Politécnica de Madrid; Universidad Carlos III de Madrid 
(3) V0070 Dripping down the rivulet.

Gaétan Lerisson, Pier Giuseppe Ledda, Gioele Balestra, François Gallaire

LFMI - EPFL

\section{Gallery video winners}

(4) V0097 Reduced adhesion of sparkling water droplets.

Divya Panchanathan, Philippe Bourrianne, Kripa K. Varanasi, Gareth H. McKinley

MIT; ETH Zurich

(5) V0057 Surfactant-free persistence of surface bubbles in a volatile liquid.

Mark Menesses, Matthieu Roché, Laurent Royon, James C. Bird

Boston University; CNRS UMR - Université Paris Diderot

(6) V0032 Nitrogen swirl: Creating rotating polygons in a boiling liquid.

Alexis Duchesne, Tomas Bohr, Barbara Bohr, Laust Tophøj

Technical University of Denmark

(7) V0027 Birth of microbubbles in turbulent breaking waves.

Wai Hong Ronald Chan, Shahab Mirjalili, Suhas S. Jain, Javier Urzay,

Ali Mani, Parviz Moin

Stanford University

Posters (5 winners: 3 Milton van Dyke and 2 Gallery Winners)

\section{Milton van Dyke Poster Award winners}

(1) P0045 Viscoelastic fishbones.

Bavand Keshavarz, Michela Geri, Gareth H. McKinley

Massachusetts Institute of Technology

(2) P0020 Effect of a wall on three-dimensionally unstable trailing vortices from a delta wing. Sarah E. Morris, C. H. K. Williamson

Cornell University

(3) P0002 Whiskey webs: Microscale "fingerprints" of bourbon whiskey.

Stuart J. Williams, Martin J. Brown VI, Adam D. Carrithers

University of Louisville

\section{Gallery Poster winners}

(4) P0056 Liquid deposition through evaporation.

Asher P. Mouat, Clay E. Wood, Justin E. Pye, Justin C. Burton

Emory University

(5) $\mathbf{P 0 0 0 4}$ Painting fluid motion using convolutional neural networks:

An album of fluid motion 2.0.

Maxime Bassenne, Andrew Banko, Sadaf Sobhani

Stanford University 